\title{
Results of application of frequency resonance therapy for treatment of cow mastitis
}

\author{
Victor E. Lyubimov ${ }^{1}$, Dmitry V. Romanov ${ }^{1}$, Yuri A. Tsoi ${ }^{1}$, Bulat G. Ziganshin ${ }^{2}$, and Farit F. Sitdikov ${ }^{3 *}$ \\ ${ }^{1}$ Federal Scientific Agroengineering Center VIM, 109428 Moscow, Russia \\ ${ }^{2}$ Kazan State Agrarian University, 420015 Kazan, Russia \\ ${ }^{3}$ Elabuga Institute of Federal State Autonomous Educational Institution of Higher Education "Kazan (Volga Region) Federal University", \\ 420008 Elabuga, Russia
}

\begin{abstract}
The physiological aspects of the implementation of the cow milk transfer reflex during machine milking are the result of exposure to the threshold force of a given value of a variable vacuum and simultaneous mechanical irritation by the teat rubber of the udder of the cow udder system. This effect leads to the discovery of sphincters with the subsequent release of the hormone oxytocin by the pituitary gland and the milk accumulation from the alveolar udder tissue. Signal environmental factors (sounds of a vacuum pump, milking machines, feeder) cause reactions in cows not only by irritation but also by frequency. It has been established that exposure to a high-frequency electromagnetic field destroys most of the common causative agents of mastitis-staphylococci, streptococci. For the treatment of mastitis, stimulation of lactation and the reflex of milk flow in cows, it is promising to apply the effect of a high frequency electromagnetic field modulated by radio frequencies, which contribute to the conversion of electromagnetic field energy into energy of nerve impulses and chemical reactions of mitochondrial cellular mechanisms of udder alveolar tissue cells. The use of alternating sets of specific frequencies of a modulating electromagnetic field of high frequency allows the alternation of "killer" frequencies for microbes with stimulating secretion of alveolar cells of the udder and with frequencies that act antiinflammatory. It is essential to determine the frequencies that create protective inhibition from exposure to extreme irritations of milking factors (increased vacuum, durable worn nipple rubber). With prolonged exposure to an electromagnetic field, such frequencies a repeating conditioned stimulus of a weak strength, which precede and accompany the impact of the milking machine, and stimulate the activity of the nerve centers of the milk yield reflex in cows, creating a dominant lactation.
\end{abstract}

\section{Introduction}

The issues of adaptation of breast tissue to the effects of the milking machine are currently insufficiently studied. It is necessary to study the mechanism of adaptation of the central nervous system of dairy cows to new industrial methods and methods of maintaining and reacting with signaling environmental factors. Cows respond not only with a specific but also with a nonspecific reaction to new milking methods, which depend on the strength of irritation and the type of nervous activity, are the most critical tasks for creating conditions for effective machine milking and prevention of mastitis. The reaction of cows depends on the strength of the stimulation and the type of nervous activity. Taking this factor into account is an essential task for creating conditions for effective machine milking and mastitis prophylaxis $[1,2]$. The system of receptors in the vessels, cisterns, excretory ducts, and skin surface of the mammary gland is designed to perceive them as mechanical stimuli, which contributes to the challenge of milk excretion and its secretion [3, 4]. The reflex of milk output can be caused both unconditionally reflexively and by the action of conditioned reflex stimuli: auditory, visual, olfactory analyzers. The complex of conditioned and unconditioned stimuli accompanying the preparation of the cow for milking, even before milking begins, causes reflex excretion of oxytocin, relaxation of the muscles of the nipple, ducts and cisterns, which ensures the transfer of milk from the alveoli to the ducts and cistern and the implementation of milk flow [4]. The problem of milk flow stimulation during machine milking has always been and is since milking machine tests are carried out without taking into account the physiological reactions of cows, but according to the final results of machine milking $[1,3,4]$.

A variety of forms of udders and nipples of different breeds of cows offers a choice. This choice is either to customize the milking machine design to the udder shape or to select cows with a suitable udder shape and size of the nipples [5]. One of the leading causes of mastitis is the different rate of quartering during machine milking due to the uneven development of the quarters, which provides increased irritation of the nipples by vacuum.

\footnotetext{
* Corresponding author: agromehanika116@gmail.com
} 
Important indicators of the suitability of cows for machine milking are the cup-shaped form of the udder, long nipples (which is ideal for milking), and the duration of issuing different quarters of the udder: most of the cows $(1.4 \%)$ have a rounded shape of the udder, $38.6 \%$ have a cup-like shape, with this, the intensity of milk transfer in cows with a cup-shaped udder is higher than in cows with a rounded shape $[2,4]$.

The main sign of latent (subclinical) mastitis - an increase in the content of somatic cells in $1 \mathrm{ml}$ of milk is continuously associated with the form of the udder and the speed of milking; despite the fact that the cow's body responds to the milking process, as a stress factor, by an increased release of leukocytes, which make up the bulk of somatic cells. The technical parameters of the milking machine do not adequately correspond not only to the modern morphology of the udder but also to the state of the general resistance of animals. Maintaining the suitability of cows for milking should have the highest priority $[1,2,4,5]$.

For the development of a milk return reflex in firstcalf heifers and new-calf cows, it is necessary to create devices adequate to the natural physiological criteria of animals to develop stable conditioned reflexes of milk return and remove the problem of manual stimulation of this return from a person $[1,4,6]$.

The use of electromagnetic fields stimulates the regulatory processes of the secretory cells of the udder themselves and the restoration of "biophysical homeostasis" disturbed by the disease [6]. Carefully selected physiologically resonant frequencies can both suppress disharmonious fluctuations that occur in affected cells during inflammation, and normalize metabolic processes, accelerating the transport of inflammatory products due to the known effect of increasing vascular permeability [7-9], while recording weak currents caused by potentials of the udder cells themselves [7, 10].

When electromagnetic fields interact with biological objects, their energy is spent mainly on healing tissues. In the low-frequency and high-frequency ranges (up to $10 \mathrm{MHz}$ ), living tissues are considered as conducting media, and the conversion of energy into heat is associated mainly with the loss of conductivity. Physical factors irritate the receptors of the skin and deep tissue. Complex reflex reactions occur, creating a long-term restructuring of the body, increasing its defenses. However, physiotherapeutic methods of treatment have contraindications for use in severe conditions of the body, inclinations to bleeding, and malignant tumors [11].

The physical side of the electromagnetic field effect on a living organism is reduced to the interaction with the electric field of the cell. This interaction is characterized by the polarization of both individual biochemical molecules and, in general, dielectric tissues, the induction of electric charges on the surface of the body, and microcurrents in conducting tissues. The electric field arising between the electrodes creates a stream of versions acting on the nerve endings in the skin as an effective biological stimulant, the effect of which is often taken as the direct action of the electromagnetic field. Therefore, exposure to electromagnetic field generators largely depends on the location of the electrodes on the irradiated organ body, i.e., from the configuration of the electric field around it [1.8]. In biomedicine, RFID (Radio Frequency Identification) systems are intensively developed and widely used. The dielectric constant of biological tissue is high at low frequencies because, at these frequencies, the membranes of bound tissue macromolecules have a larger capacity.

At frequencies above $100 \mathrm{MHz}$, the peculiarity of the membrane capacitance due to the rotational and vibrational properties of polar water molecules leads to a decrease in the dielectric constant of the biological medium and an increase in conductivity with increasing frequency [12]. The body structures are sources of lowintensity electromagnetic radiation (power and tension) both by themselves and with a specific external excitation. Based on this principle, such diagnostic methods as electroencephalography, electrocardiography, and other methods that use low current loads are used: the Nakatani-mA method, the Voll method $-\mu \mathrm{A}$, the Schimmel method (vegetative resonance or bioresonance test).

Cell organelles, cells, and tissues are characterized by both general and specific, differing electromagnetic characteristics, have their inherent spectra and radiation and absorption, are low-intensity EMF sources, forming conductive or oscillatory circuits and can be described from the perspective of electromagnetic interactions. An example of this is the well-known Nernst equation for determining mitochondrial potentials.

\section{Materials and methods}

The device we developed uses amplitude modulation of the electromagnetic field of high frequency. This modulation stimulates biological responses in the body of cows by creating physical resonance in cellular organelles, cells, and animal tissues. Electromagnetic resonance in the biological systems of living organisms leads to the emergence of the effects of conditionally "biological resonance". Changes in the resonance "amplitudes" in the vibrational electromagnetic circuits of cells lead to changes in ionic strength, ion transport intensity in media, membrane permeability, and metabolic processes (biochemical reactions). These changes are implemented both at the level of cellular organelles and cells and the level of tissues, organs, and the whole organism. Effects can be regulatory, treatment-stimulating, or killer. The goal of our research is to determine modulating frequencies that are fatal for possible pathogens that cause mastitis and frequencies that restore the secretion of alveolar cells. We evaluate the effects of the presence of "biological resonance" by clinical signs and by laboratory analysis of the content of somatic cells in milk samples. The analysis includes a comparison of clinical signs before and after exposure to an electromagnetic field. In this way, a secondary analysis based on the micro reaction of the cow's body is possible. 
The radio frequencies modulate a carrier frequency of $27.0 \mathrm{MHz}$ in series with a period equal to the specified exposure time of each frequency. The output electromagnetic radiation is a meander with a duty cycle of 0.5 , depth of amplitude modulation of $100 \%$, and a generation accuracy of $0.01 \mathrm{~Hz}$.

Highly specific reactions or bio-responses to an external modulated low power electromagnetic field are known. In the application of a high-frequency electromagnetic field, there are differences in the nominal frequencies of the carrier frequencies, sets of FA modulating frequencies, electromagnetic parameters (intensity, power, pulse type, duty cycle), but only in the general circuit diagram, individual elements and their design [7-9]. Exposure in the near zone (at a distance shorter than the wavelength) can cause effects that depend more on the frequency (wavelength) and less on the radiation power.

Physiotherapy using UHF devices (according to the international classification it is $\mathrm{HF}$, because less than 30 $\mathrm{MHz}$ ) at a frequency of $27.12 \mathrm{MHz}$ (wavelength 11.02 M) from the allowed range of 27.0-27.2 MHz, and at a frequency of $40.65 \mathrm{MHz}$ (the wavelength of $7.35 \mathrm{~m}$ ) is carried out in accordance with the developed methodological recommendations for the location of the emitting electrodes at distances from $0.5 \mathrm{~cm}$ or more (up to several $\mathrm{cm}$ ). The application of the influence of an electromagnetic field of more or less of these frequencies (going beyond the frequency range) leads to the loss of biological effects. These biological effects are recorded upon exposure to an electromagnetic field in the near zone.

The effect of the electromagnetic field is reduced to:

- interaction with the electric field of the cell;

- polarization of both individual biochemical molecules and dielectric tissues as a whole;

- to induce electric charges on the surface of the body, and microcurrents in conductive tissues.

The emerging electric field creates a stream of ions acting on the nerve endings in the skin as an effective biological stimulant, the effect of which is often taken as the direct action of the electromagnetic field. Therefore, the effect of electromagnetic field generators largely depends on the location of the electrodes on the irradiated organ object, i.e., from the configuration of the electric field around it. The influence of an external electromagnetic field on the body consists of accelerating or slowing down biochemical and biophysical processes. But the cells do not passively "execute" the control of the external electromagnetic field, but actively interact (with each other) with their electromagnetic field.

The source of all cellular oscillations is the free energy produced by metabolic processes. A cell can be considered as a micropower "electromechanical generator" with a molecular mechanism for using the energy of the electromagnetic field and the energy of chemical bonds in its life [10]. This mitochondrial mechanism converts the energy of an electromagnetic field into an electric current (nerve impulse), into the energy of a chemical reaction and the energy of an electromagnetic field (synapse) [7]. The external electromagnetic field interacts not only with the cell's fields. The fields of the cells interact with each other, and the interaction of the fields is possible only if the cell itself as a generator is resonant with the system of interacting electromagnetic fields.

The influence of an external electromagnetic field of high frequency resonantly enhances the generation by the cell at the frequency of the "pathology" of natural oscillations, fueled by an external electromagnetic field, forms, and includes its generating system. This generating system:

a) it "draws in" the influx of free energy of metabolism to a given frequency spectrum of cell oscillations;

b) it initiates the generation of vibrations in this (narrow) spectrum of healthy cells of localized aggregation, the mechanism of chemotaxis is activated, and the direction of biochemical processes changes towards normalization of the cell [10].

Experiments on animals and observing people during physiotherapy reflect its undoubted effect on many functions of living organisms. In the interaction of the electromagnetic field with biological objects, the field energy is mainly spent on heating the tissues. In the lowfrequency and high-frequency ranges (up to $10 \mathrm{MHz}$ ), almost all body tissues are like conductive media, and the conversion of energy into heat is associated mainly with the loss of conductivity.

The electromagnetic fields of ultra-high and high frequencies lead to irritation of the receptors of the skin and deep-seated issues. In response to these irritations, complex reflex-type reactions occur that cause a prolonged restructuring of the body. As a result, the animal's defenses increase. However, physiotherapeutic methods of treatment have contraindications for severe conditions of the body, a tendency to bleeding, and malignant tumors $[6,10,11]$.

Cows, for many decades, have been selected for high milk production. As a result of such selection, highly milk cows lose their ability to maintain the necessary level of factors of natural defense and balance of the internal environment - homeostasis. The technology of "voluntary milking" with free housing compared to tethered is accompanied by a stronger activation of the processes of excitation of neurons of the lactational and other nerve centers of conditioned and unconditioned reflexes. Cow organisms do not have time to develop appropriate reactions (including protective ones) to new technological principles introduced into milk production to reduce labor costs and maximize economic benefits.

Adaptation of cows to conditions of loose housing with the help of analyzers and activation of the endocrine glands that accelerate metabolic processes (according to the doctrine of the general adaptation syndrome of G. Selye) really represents the transfer of the cow's organism from the state of the "anxiety" stage to the state of "increased resistance". With free housing, cows are affected by various stress factors: a struggle for a place at the feed table, pain from crawling milking cups as they are milked and "dry milked," the tightness of being in front of the milking parlor when the decrease 
in individual distances provokes aggressive behavior of animals. Therefore, most cows experience emotional stress, accompanied by an increase in the tone of the sympathoadrenal system with an increase in the concentration of catecholamines in the blood.

With increasing exposure to stress factors, for example, over-threshold stimulation of the udder receptors by milking factors increased flows of afferent excitation inform the central nervous system of aggression. As a result, the brain of cows activates the somatomotor, visceromotor, and endocrine systems. In the hypothalamus, releasing factors that stimulate the formation of hormones of the anterior pituitary gland, which activate metabolic processes, and especially the adrenocorticotropic hormone $\mathrm{ACTH}$, begin to stand out more.

An increased concentration of ACTH in a cow is determined in an anxiety reaction state when all adrenal cortex is activated. ACTH stimulates the release of catecholamines, and they always mobilize the breakdown of glycogen and increase the concentration of sugar in the blood, creating the conditions for the transition of the cow's body to a state of increased general resistance $[3,13]$. The increase in milk secretion in the first 100 days of lactation often leads to a negative energy balance, a decrease in fat layers, and insufficient secretion of the endocrine glands.

It is known that the concentration of mechanoreceptors in the area of the base of the udder nipple exceeds the number of mechanoreceptors in the apex and middle part of the udder nipple, and increases sharply from 354 to 2477 and/s with increasing concentration of oxytocin in the blood. It has been established that an increase in the biologically active area points at the base of the nipple occurs during milk flow and inflammation of the udder with mastitis, and during recovery, the area of the point returns to its original state [14].

Studies of the effects of electric current on dogs by I.P. Pavlov proved that the obtained effects of weak and strong stimuli reflect the general reaction of the body: irritation of nerve endings, skin receptors, and internal organs excites the central nervous system and neurons of the cerebral cortex, which changes metabolism and blood circulation.

Under the influence of an electromagnetic field of ultrahigh-frequency (30-300 MHz), weak doses increase the function of tissues and organs, and strong ones suppress them. The activity of phagocytes increases, active hyperemia intensifies, swelling of tissues decreases, and the sensitivity of nerve endings decreases $[6,8,9,11]$.

Mastitis is inflammation of the tissues of the udder and nipple with a primary lesion of the epithelium of the mucous membrane of the milk tank and milk ducts. Under the influence of intoxication, the accumulation of microbial toxins increases the irritation of nerve endings. The inflammation development is determined by the cow's defenses state, as well as the pathogen pathogenicity.

When the defenses are weak, then mastitis takes a malignant course and causes a septic state. With the development of the inflammatory process in the affected tissues of the udder, significant morphological changes appear. Morphological changes appear in the interstitial tissue in the form of diffuse cellular infiltrates, a decrease in the size of the alveoli. Casein clots and flakes appear in the milk. The number of leukocytes in milk increases 5-10 times [15].

Each cow individually responds to machine milking and has its threshold for reflex sensitivity and reactivity of its nervous system. It has been established that the main factor providing machine milking with minimal disturbance in the physiology of the milk transfer reflex is the preparation for connecting the milking machine with mandatory 40 -second stimulation, i.e., compliance with the rules of machine milking [4]. With regular milking, irritation with vacuum and nipple rubber increases the sensitivity of the nipples, which, on the one hand, makes it milk, and on the other, it creates a feeling of pain, and the cows are forced to endure milking. It is known that the best milk yield is caused by mechanical irritation of the udder with an intensity of $2-2.2 \mathrm{~kg} / \mathrm{s}$, lasting $40 \mathrm{~s}$ [16].

The formation of the stereotype of the milk transfer reflex, adaptation to the milking unit, to the milking conditions in the milking machine, according to the development of the general theory of I.P. Pavlova, is an animal teaching. The transition to new technology for keeping dairy cows (loose) is accompanied by fundamental changes in the field of the psyche of animals: a new circadian rhythm is created, and the individuality of animals is more pronounced. Both the general excitability of animals and the sensitivity of receptor systems increase.

In order to withstand daily threshold irritation of udder receptors for several months, the cow's body mobilizes reserves of natural resistance, and a group of inhibitory neurons is formed between the centers of the conditioned and unconditioned reflexes in the centers of the central nervous system, along with the formation of a "temporary connection" with the formation of the shortest path of excitation. Renshaw, providing protective inhibition of the presynaptic type, preserving the physiological threshold of excitability of the nervous system for successful re laicization of the dominant lactation and reflex of milk flow.

The milking process can be considered as a natural physiological test to determine the tone of the autonomic nervous system. It is known that an increased vacuum in the suction cup of the teat cup causes Vibro-injury to the nipple of the mammary gland, which leads to a change in the electroencephalogram in the form of desynchronization [1].

In industrial conditions, in the range of milk flow rates from 1 to $2.8 \mathrm{l} / \mathrm{min}$. from one quarter, the average vacuum level in the nipple chamber decreases even more significantly - by $3-6 \mathrm{kPa}$. These differences are caused by cow moving during milking, not only when uncontrolled air intake around the nipples occurs, but more often when there is pain from the "creeping" milking cups onto the nipples. Often the reason for the cessation of lactation is the reflex response of the central nervous system to the systematic pain of the mammary 
gland with improper milking. It was found that a strong vacuum (more than $380 \mathrm{~mm} \mathrm{Hg}$ ) is the cause of pain irritation of the receptors of the sphincter of the nipple and the entire mammary gland. But what threshold values have udder nipple receptors in lactating cows has not yet been clarified. Exterior- and interceptors of the mammary gland are not irritated simultaneously and have different thresholds of excitability. A large percentage of culling due to early mastitis depends on the incorrect selection of the vacuum regime, which is especially difficult for the state of the udder receptor system during overexposure of teat cups $[2,14]$.

\section{Results}

The medical device "Akutest" is intended for diagnostics and frequency resonance therapy. It is applied for conducting electromagnetic effects at specific resonant frequencies to provide a general regulatory effect on the physiological systems and organs of a mammalian organism in a wide range of pathogens studied (microbes, viruses, helminths, and other parasites). In medicine, combined devices are used that combine the functions of a communicator (iPhone or smartphone) and electromagnetic therapy devices to stimulate the body with a weak low-frequency modulated electromagnetic field to treat various diseases and increase the body's defenses [4, 17].

For long-term exposure to a dairy farm, we have improved the Akutest apparatus. Improvement of the Akutest apparatus was carried out in the following areas. We developed and manufactured a sealed shockproof housing and added a USB channel to the generator design to load exposure modulation programs and a communication channel with a milking station controller system, which allows controlling and monitoring the operation of the high-frequency electromagnetic field generator remotely through Wi-Fi system.

An indicator of recovery of the udder of a cow is the components of the secretion of the udder: the number of somatic cells, organoleptic components of the secret (blood, cereal, milk uniformity, and other indicators). Frequency modulating electromagnetic fields do not force the cells of the tissues and organs of the cow to work in the mode in which they are not able to work as a result of the effects of mastitis and the pathological changes caused by it - the inflammatory process and the resulting immune reactions.

Frequency modulating electromagnetic fields create the conditions for restoring the health of the secretory cells of the udder (generating potentials that secrete the secretion of milk components). In medical practice, when exposed to frequency resonance therapy of various intensities, the absence of pain during sessions of different durations was established. For the treatment of various diseases of people, the optimal time (duration) of exposure to one selected and programmed frequency is 5 minutes. The effect of electromagnetic therapy in a person does not appear immediately, but after a few days [4, 7, 8-10, 18].

The use of an autonomous medical device "LORZ" with exposure to a modulated electromagnetic field of high frequency is painless for cows and does not cause a defensive reaction. A device developed by us for the treatment of mastitis in cows - a high-frequency electromagnetic field generator in the applicator, provides a long-term effect (from 24 to 96 hours) on the cow.

The duration of exposure depends on the restoration of milk secretion in the quarter affected by mastitis. The effect of an electromagnetic field of ultrahigh and high frequency adopted in medical therapy was used. It has been established that heating of bone, muscle, and adipose tissue due to the electromagnetic field of ultrahigh and high frequency occurs more intensively than the heating of blood vessels and lymph nodes. The action of the ultra-high frequency electromagnetic field is pronounced deep in the tissues where there is an increase in the activation of enzymes, the transformation of coarse protein molecules into smaller ones with a change in the $\mathrm{pH}$ of the cytoplasm.

The self-renewable generator battery of the LORZ device forms a modulated electromagnetic field of high frequency. They do not cause heating of tissuedielectrics but cause the cells of secretory tissues and the circulatory system to adapt to the modulating frequencies of an external electromagnetic field. As a result, the effect of the device restores the reduced electrical activity of cells affected by mastitis tissue. The generation of the device action potentials forms its electromagnetic field in the form of an excitation process with an increase in the level of secretion of the corresponding components.

The therapeutic effect of exposure to a high-frequency electromagnetic field on parts affected by mastitis consists of both suppressing the activity of known pathogens and normalizing inflammatory reactions and stimulating the restoration of the secretory activity of alveolar cells. Cows, when exposed to a high-frequency electromagnetic field by an autonomous device, move freely in sections. Animals feed, drink, lie, come to the milking, become in the milking machine of the milking machine. In the developed LORZ device, the exposure modulation program serves to prevent and treat udder diseases, stimulate lactation of cows, USB channel in the generator. The communication channel with the system of controllers of milking places in the LORZ device allows controlling and monitoring the operation of the highfrequency electromagnetic field generator remotely.

Milking is carried out, taking into account the results of preparing for milking and assessing the condition of the udder, followed by monitoring the results of milking. The machine milking operator can enter data on the condition of the udder affected by mastitis through the button system of the milking controller "M," "M1," and "M." Most modern farms are equipped with a router for distributing the Internet via a $\mathrm{Wi}-\mathrm{Fi}$ radio channel through which you can receive data from milking point controllers. Routers allow controlling the operation of an electromechanical device. The channel of the Wi-Fi radio module in the device allows the veterinarian remotely changing the parameters of the treatment program for exposure to the high-frequency 
electromagnetic field through the controller of the milking place.

In this case, the veterinarian can perform these operations without removing the milking device from the cow. The veterinarian can remotely increase or decrease the duration of treatment-exposure according to the results of assessing the condition of the affected quarter during the current milking by the machine milking operator. The Wi-Fi system allows controlling the operation of the generator. The antenna identifies the cow number by the collar transponder or by the pedometer at the milking unit entrance.

The cow gets into the milking machine, and its identified number is displayed in the controller of the milking place. The machine milking operator the first trickles off and checks the condition of the applicator and the operation of the generator in the process of premilking prepares. If there are signs of inflammation of the udder ("crickets," flakes, pus, blood), the operator presses the "M" button in the controller and connects the milking machine to a separate tank for mastitis milk.

Table 1. Change in the number of somatic cells in milk after exposure to a high frequency modulated electromagnetic field (frequency resonance therapy)

\begin{tabular}{|c|c|c|c|c|c|c|c|c|}
\hline № & $\begin{array}{c}\text { Cow } \\
\text { number }\end{array}$ & $\begin{array}{l}\text { The number of } \\
\text { somatic cells, } \\
\text { (x 106). Source }\end{array}$ & $\begin{array}{c}\text { The number of } \\
\text { somatic cells, (x 106). } \\
\text { After treatment in } 13 \\
\text { days }\end{array}$ & $\begin{array}{l}\text { The degree of } \\
\text { mastitis in keno } \\
\text { test for all "+" } \\
\text { BEFORE }\end{array}$ & $\begin{array}{c}\text { Number } \\
\text { of } \\
\text { sessions }\end{array}$ & $\begin{array}{c}\text { Keno test } \\
\text { mastitis degree } \\
\text { for all "+" } \\
\text { AFTER }\end{array}$ & $\begin{array}{c}\text { After / } \\
\text { before ratio }\end{array}$ & $\begin{array}{l}\text { After / before } \\
\text { ratio }\end{array}$ \\
\hline 1 & 21050 & 3,046 & $<3,0$ & 4 & 3 & 2 & & \\
\hline 2 & 10254 & 2,499 & $<3,0$ & 3 & 3 & 1 & & \\
\hline 3 & 12412 & 3,259 & $<3,0$ & 3 & 3 & 1 & & \\
\hline 4 & 5423 & 3,83 & $<3,0$ & 5 & 3 & 1 & & \\
\hline 5 & 5430 & 9,02 & $<3,0$ & 6 & 5 & 3 & & \\
\hline 6 & 3854 & 5,01 & 2,544 & 5 & 2 & 2 & 0,508 & 1,969 \\
\hline 7 & 11584 & 5,07 & 3,56 & 6 & 3 & 3 & 0,702 & 1,424 \\
\hline 8 & 10360 & 2,188 & 1,123 & 7 & 5 & 4 & 0,513 & 1,948 \\
\hline 9 & 12031 & 2,305 & 0,924 & 5 & 5 & 3 & 0,401 & 2,495 \\
\hline 10 & 1130 & 5,0001 & 2,807 & 4 & 5 & 1 & 0,561 & 1,781 \\
\hline 11 & 13026 & 9,87 & 3,2 & 8 & 5 & 4 & 0,324 & 3,084 \\
\hline 12 & 14008 & 3,467 & 1,84 & 8 & 5 & 3 & 0,531 & 1,884 \\
\hline 13 & 10261 & 3,985 & 0,97 & 4 & 3 & 1 & 0,243 & 4,108 \\
\hline 14 & 5624 & 6,25 & 0,12 & 3 & 3 & 1 & 0,019 & 52,083 \\
\hline 15 & 12299 & 4,258 & 0,117 & 9 & 3 & 3 & 0,027 & 36,393 \\
\hline 16 & 5324 & 2 & 5,537 & 5 & 3 & 4 & 2,769 & 0,361 \\
\hline 17 & 14028 & 1,9 & 2,934 & 8 & 5 & 6 & 1,544 & 0,648 \\
\hline & \multicolumn{7}{|c|}{ Average value } & 9,015 \\
\hline
\end{tabular}

Note: cows No. 5324 and No. 14028 had advanced chronic purulent-catarrhal mastitis.

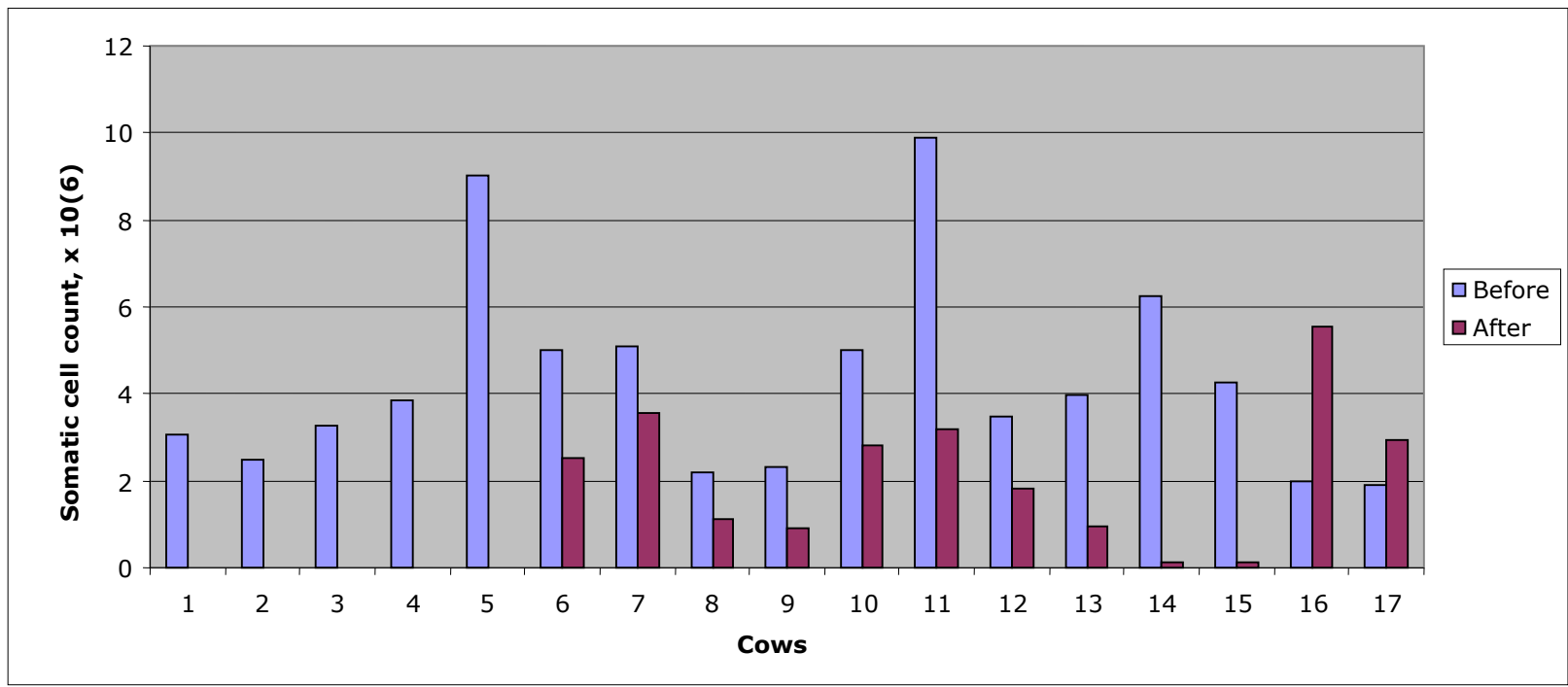

Fig. 1. Change in the number of somatic cells in milk after exposure to a high-frequency electromagnetic field (frequency resonance therapy) 
The veterinarian receives a signal to the smartphone from the milking controller and remotely checks the battery charge, duration (session duration), and a number of the set frequency program. According to the test results for the treatment of mastitis, 8 developed and tested programs that modulate the electromagnetic field of a high frequency in the range of $1-700000 \mathrm{~Hz}$, which inhibit possible pathogens, were experimentally selected. The specialist can remotely change the alternating order of a set of modulating frequencies using a Wi-Fi system.

17 cows with clinical and subclinical signs of mastitis (according to keno-test) were treated. Clinical signs (soreness of the nipples of the udder, blood, and flakes in the milk, "crickets") were identified together with the state farm veterinarians. Samples of milk for the number of somatic cells were taken before treatmentexposure with a high frequency modulated electromagnetic field by the LORC device and after the veterinarian established the recovery of the affected quarter by the veterinarian. 68 treatment sessions were conducted with a duration of 24 to 48 hours of continuous exposure, depending on the disappearance of signs of mastitis. Milk samples were examined to calculate the number of somatic cells in milk in the regional veterinary laboratory.

3 cows finally recovered. 12 animals showed a significant improvement in the state of the udder.

\section{Conclusion}

The provisional application of specific resonant frequencies of modulation of the electromagnetic field of a high frequency in the form of a programmed set of selected and established resonant frequencies contributed to the treatment of mastitis affected quarters of cows. Resonant frequencies modulate the electromagnetic field of high frequency, inhibiting the activity of known pathogens. The applied set of frequencies relieves inflammation and stimulates the restoration of regular secretory activity of alveolar cells. An autonomous device for the mastitis treatment does not interfere with the cow in all its physiological functions. An autonomous device does not interfere with machine milking both in the milking parlor and during milking by the robot. At the same time, the device is reliable in operation and affordable for the rural veterinarian. The purchase price of the device pays for the effectiveness of the therapeutic effect. The power supply of an electromechanical device in an industrial dairy farm is autonomous and safe.

The high frequency electromagnetic field generator in the applicator can be on the cow for a long time (from 24 to 96 hours) depending on the restoration of milk secretion in the quarter of the cow's udder affected by mastitis. The duration of exposure is the novelty of applying exposure to modulated physiologically resonant frequencies of the high-frequency electromagnetic field of an autonomous device for treating mastitis in cows.

The development and application of an autonomous therapeutic device for prolonged exposure to a modulated electromagnetic field is an engineering solution to the problem of treating nonspecific mastitis in cows on an industrial dairy farm under conditions of free housing.

The implementation of this concept is an energysaving and environmentally friendly and electrically safe technology. Frequency-resonant exposure to a highfrequency electromagnetic field on mast udder of cows in an industrial dairy farm can be carried out both with free and tethered methods of keeping cows. It is possible to create a device controlling the intensity of the electromagnetic field exposure on a robotic milking machine.

When the electromagnetic field frequencies are ultrahigh and high frequencies adopted in medical therapy, the heating of bone, muscle, adipose tissue occurs more intensively than the heating of blood vessels, lymph nodes, and other parts. The action of ultra-high frequencies is pronounced in deeply lying tissues, where there is an increase in the activation of enzymes, the transformation of coarse protein molecules into smaller ones with a change in the $\mathrm{pH}$ of the cytoplasm. The therapeutic use of the field of ultra-high frequencies should be carefully dosed since it has a strong physiological effect $[1,11,17,18]$.

The LORZ device generates modulated frequencies of a high-frequency electromagnetic field. The therapeutic effect of the LORZ device does not cause intense heating of tissue-dielectrics. However, the effect of the LORZ device causes the cells of the primary secretory tissues and the circulatory system to adapt to the physiological frequencies of the external electromagnetic field. As a result, cells restore and generate their electromagnetic fields, corresponding to their vital action potentials, with an increase in the level of secretion of the corresponding components. The therapeutic effect of exposure to a high-frequency electromagnetic field on mast quarters of cows udders is to suppress the activity of known pathogens and to relieve inflammation and stimulate the restoration of regular secretory activity of alveolar cells.

\section{References}

1. A.N. Golikov, E.I. Lyubimov, New in the physiology of the nervous system of farm animals (Kolos, Moscow, 1977)

2. O.V. Gorelik, O.A. Vagapova, Milk productivity, its relationship with the form of the udder and the intensity of milk yield in black and white cows, in Proc. of the 10th Int. Symp. on Milking Agricult. Animals, 225-228 (2002)

3. V.F. Korolev, Milking machines, 2rd ed. (Engineering, Moscow, 1969)

4. E.P. Kokorina, Conditioned reflexes and animal productivity (Nauka, Moscow, 1986)

5. K. Count, Sh. Heidel, G. Svalve, W. Bergfeld, The Close Connection of Form and Functionality, New Agricult., 1, 84-86 (2007)

6. V.E. Lyubimov, The influence of electromagnetic fields of ultrahigh-frequency on the mammary gland 
of cows during machine milking, $\mathrm{PhD}$ dissertation thesis (Moscow veter. academy named after K.I. Skryabin, Moscow, 2004)

7. E.V. Teslin, Classical bio-resonance therapy, according to F. Morell, Educational-methodical manual (Tokran Corporation, St. Petersburg, 2013)

8. Device for electromagnetic therapy. RF patent no. 109412, IPC A61N2 / 04 (publ. 20.10.2011).

9. The method and therapeutic device for exposure to a pulsed frequency resonance electromagnetic field for the prevention and treatment of diseases of the udder of cows, stimulation of lactation. R.F. patent for invention no. 2644826 (publ. 02.14.2018. Bull. No. 5).

10. T.I. Subbotina, A.A. Yashin, Resonance effects in the interaction of electromagnetic fields with biosystems. Part II, Bull. of new med. technol., 12(4), 152-171 (2018)

11. A.R. Livenson, Electromedical equipment, 5rd ed. (Medicine, Moscow, 1981)

12. I.B. Vendik, D.S. Vendik, D.S. Kozlov et al., Wireless monitoring of the state parameters of biological objects in the microwave range, $\mathrm{J}$. of Techn. Phys., 86(1), 3-26 (2016)
13. M. Kovalchikova, K. Kovalchik, Adaptation and stress during the maintenance and breeding of farm animals (Kolos, Moskow, 1978)

14. R.I. Korneeva, Electrophysiological analysis of the receptive function of the udder, $\mathrm{PhD}$ dissertation thesis (Borovsk, 1986)

15. V.A. Akatov, G.A. Kononov, A.I. Pospelov, I.V. Smirnov, Veterinary obstetrics and gynecology: Textbook for high schools (Kolos, Moscow, 1977)

16. S. Israilzhanov, A. Averkiev, Dependence of milk yield on the intensity and duration of breast stimulation, in Mater. of the 4th All-Union Symp. on the physiolog. Foundat. of machine milking, 100 (Almaty, 1975)

17. T.V. Ippolitova, Physiological parameters of the central, nervous, sympathoadrenal, and cardiovascular systems of cows in the process of adaptation to industrial technology, $\mathrm{PhD}$ dissertation thesis (Moscow veter. academy named after K.I. Scriabin, Moscow, 1992)

18. A.S. Belanovsky, Fundamentals of biophysics in veterinary medicine (Bustard, Moscow, 2007) 